Supporting Information

\title{
Novel Approach to the Analysis of Chemical Third-Order Data
}

Shao Hua Lu, Hong Lin Zhai*, Bing Qiang Zhao, Bo Yin, Ling Zhu

College of Chemistry \& Chemical Engineering Lanzhou University, Lanzhou 730000, PR China

The corresponding author's e-mail address: zhaihl@163.com

\section{The experimental and calculated concentrations $(\mu \mathrm{M})$}

Table S1. The experimental (Exp.) and calculated (Cal.) concentrations $(\mu \mathrm{M})$ of the two components in Data I (TCM models)

\begin{tabular}{|c|c|c|c|c|c|c|c|c|c|}
\hline \multirow{3}{*}{$\begin{array}{l}\text { Sample } \\
\text { no. }\end{array}$} & & \multicolumn{4}{|c|}{ Adrenaline } & \multicolumn{4}{|c|}{ Noradrenaline } \\
\hline & & \multirow{2}{*}{ Exp. } & \multicolumn{3}{|c|}{ Cal. } & \multirow{2}{*}{ Exp. } & \multicolumn{3}{|c|}{ Cal. } \\
\hline & & & $\mathrm{X} 20$ & X60 & Xlarge & & $\mathrm{X} 20$ & X60 & Xlarge \\
\hline Calibration & 1 & 0.50 & 0.54 & 0.53 & 0.53 & 0.00 & 0.01 & 0.01 & -0.01 \\
\hline set & 2 & 0.50 & 0.49 & 0.49 & 0.51 & 0.00 & 0.02 & 0.00 & -0.01 \\
\hline & 3 & 0.00 & 0.00 & 0.01 & 0.00 & 1.00 & 0.98 & 0.99 & 0.99 \\
\hline & 4 & 0.00 & 0.01 & 0.00 & 0.01 & 1.00 & 0.94 & 0.95 & 0.95 \\
\hline & 5 & 0.25 & 0.22 & 0.23 & 0.24 & 0.25 & 0.24 & 0.25 & 0.25 \\
\hline & 6 & 0.25 & 0.24 & 0.24 & 0.25 & 0.25 & 0.26 & 0.26 & 0.26 \\
\hline & 9 & 0.00 & 0.00 & -0.01 & -0.02 & 0.60 & 0.58 & 0.58 & 0.59 \\
\hline & 1 & 0.00 & 0.02 & 0.04 & -0.01 & 0.60 & 0.62 & 0.63 & 0.63 \\
\hline & 1 & 0.05 & 0.08 & 0.08 & 0.06 & 0.10 & 0.11 & 0.11 & 0.12 \\
\hline & 1 & 0.05 & 0.06 & 0.05 & 0.06 & 0.10 & 0.09 & 0.10 & 0.09 \\
\hline & 1 & 0.10 & 0.11 & 0.12 & 0.10 & 0.30 & 0.30 & 0.31 & 0.31 \\
\hline & 1 & 0.10 & 0.09 & 0.10 & 0.11 & 0.30 & 0.31 & 0.31 & 0.32 \\
\hline & 1 & 0.40 & 0.40 & 0.40 & 0.40 & 0.70 & 0.72 & 0.73 & 0.71 \\
\hline & 1 & 0.40 & 0.39 & 0.38 & 0.39 & 0.70 & 0.71 & 0.72 & 0.70 \\
\hline & 1 & 0.50 & 0.50 & 0.51 & 0.54 & 0.20 & 0.18 & 0.19 & 0.19 \\
\hline & 2 & 0.50 & 0.50 & 0.49 & 0.49 & 0.20 & 0.20 & 0.21 & 0.19 \\
\hline & 2 & 0.35 & 0.32 & 0.34 & 0.34 & 0.70 & 0.70 & 0.70 & 0.69 \\
\hline & 2 & 0.35 & 0.35 & 0.34 & 0.33 & 0.70 & 0.73 & 0.71 & 0.72 \\
\hline & 2 & 0.40 & 0.39 & 0.40 & 0.40 & 0.40 & 0.41 & 0.41 & 0.41 \\
\hline & 2 & 0.40 & 0.39 & 0.38 & 0.39 & 0.40 & 0.40 & 0.40 & 0.40 \\
\hline & 2 & 0.20 & 0.21 & 0.21 & 0.21 & 0.50 & 0.51 & 0.51 & 0.51 \\
\hline & 2 & 0.20 & 0.19 & 0.20 & 0.21 & 0.50 & 0.54 & 0.52 & 0.52 \\
\hline & 2 & 1.00 & 0.92 & 0.94 & 0.90 & 0.00 & -0.02 & -0.02 & -0.03 \\
\hline & 2 & 0.00 & 0.01 & 0.01 & 0.01 & 0.30 & 0.29 & 0.28 & 0.29 \\
\hline & 3 & 0.00 & 0.00 & 0.00 & -0.01 & 0.30 & 0.27 & 0.29 & 0.29 \\
\hline & 3 & 0.00 & 0.00 & -0.01 & 0.00 & 1.40 & 1.42 & 1.41 & 1.39 \\
\hline & 3 & 0.80 & 0.76 & 0.77 & 0.79 & 0.00 & -0.01 & -0.02 & 0.00 \\
\hline
\end{tabular}




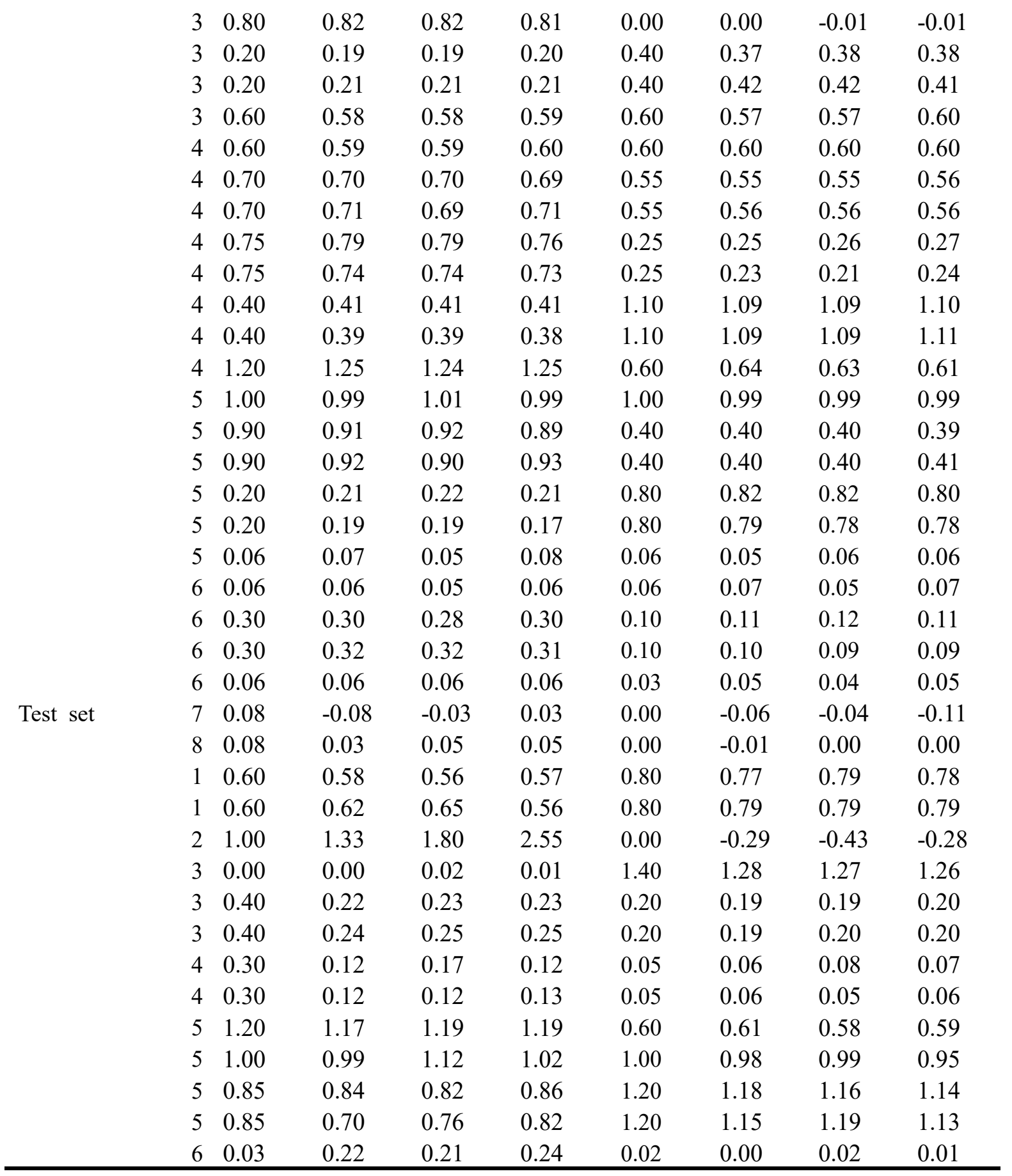


Table S2. The experimental (Exp.) and calculated (Cal.) concentrations ( $\mu \mathrm{g} / \mathrm{mL})$ of the five components in Data II (TCM models).

\begin{tabular}{|c|c|c|c|c|c|c|c|c|c|c|}
\hline \multirow{2}{*}{$\begin{array}{l}\text { Sample } \\
\text { no. }\end{array}$} & \multicolumn{2}{|c|}{ Val-Tyr-Val } & \multicolumn{2}{|c|}{ Trp-Gly } & \multicolumn{2}{|c|}{ Phe } & \multicolumn{2}{|c|}{ Malto } & \multicolumn{2}{|c|}{ Propanol } \\
\hline & Exp. & Cal. & Exp. & Cal. & Exp. & Cal. & Exp. & Cal. & Exp. & Cal. \\
\hline \multicolumn{11}{|c|}{ Calibration set } \\
\hline 1 & 5.00 & 4.87 & 0.00 & -0.04 & 0.00 & 0.04 & 0.00 & 0.01 & 0.00 & -0.05 \\
\hline 2 & 0.00 & -0.09 & 5.00 & 4.78 & 0.00 & -0.09 & 0.00 & 0.01 & 0.00 & 0.02 \\
\hline 3 & 0.00 & 0.16 & 0.00 & 0.06 & 5.00 & 5.19 & 0.00 & 0.00 & 0.00 & 0.00 \\
\hline 4 & 0.00 & 0.05 & 0.00 & 0.29 & 0.00 & -0.02 & 5.00 & 5.04 & 0.00 & 0.11 \\
\hline 5 & 0.00 & -0.04 & 0.00 & 0.14 & 0.00 & -0.05 & 0.00 & -0.08 & 5.00 & 4.93 \\
\hline 6 & 1.25 & 1.32 & 5.00 & 5.12 & 3.75 & 3.72 & 3.75 & 3.74 & 0.00 & -0.03 \\
\hline 7 & 3.75 & 3.67 & 1.25 & 1.24 & 5.00 & 4.92 & 1.25 & 1.24 & 0.00 & -0.03 \\
\hline 8 & 2.50 & 2.60 & 5.00 & 5.13 & 2.50 & 2.46 & 1.25 & 1.24 & 0.00 & 0.02 \\
\hline 9 & 5.00 & 5.06 & 3.75 & 3.79 & 2.50 & 2.66 & 3.75 & 3.64 & 0.00 & 0.01 \\
\hline 10 & 3.75 & 3.64 & 3.75 & 3.83 & 5.00 & 4.85 & 0.00 & 0.10 & 1.25 & 1.25 \\
\hline 11 & 6.25 & 6.49 & 1.25 & 1.24 & 1.25 & 1.25 & 0.00 & 0.03 & 2.50 & 2.55 \\
\hline 12 & 1.25 & 1.19 & 5.00 & 4.60 & 2.50 & 2.77 & 0.00 & -0.06 & 5.00 & 5.05 \\
\hline 14 & 5.00 & 4.98 & 1.25 & 1.46 & 3.75 & 3.75 & 1.25 & 1.26 & 3.75 & 3.74 \\
\hline 15 & 1.25 & 1.27 & 3.75 & 3.52 & 2.50 & 2.52 & 2.50 & 2.52 & 2.50 & 2.52 \\
\hline 16 & 3.75 & 3.65 & 3.75 & 3.55 & 1.25 & 1.23 & 5.00 & 5.07 & 1.25 & 1.26 \\
\hline 17 & 2.50 & 2.54 & 3.75 & 4.26 & 1.25 & 1.11 & 1.25 & 1.26 & 6.25 & 6.21 \\
\hline 18 & 1.25 & 1.26 & 0.00 & -0.07 & 5.00 & 4.88 & 2.50 & 2.47 & 0.00 & -0.03 \\
\hline 22 & 3.75 & 3.72 & 0.00 & -0.13 & 3.75 & 3.69 & 0.00 & 0.03 & 5.00 & 5.12 \\
\hline 25 & 2.50 & 2.53 & 0.00 & -0.03 & 2.50 & 2.62 & 0.00 & 0.00 & 1.25 & 1.22 \\
\hline 26 & 5.00 & 4.99 & 0.00 & -0.16 & 1.25 & 1.30 & 3.75 & 3.70 & 3.75 & 3.65 \\
\hline 27 & 3.75 & 3.63 & 0.00 & -0.08 & 5.00 & 4.95 & 2.50 & 2.52 & 1.25 & 1.24 \\
\hline \multicolumn{11}{|c|}{ Test set } \\
\hline 13 & 2.50 & 2.74 & 6.25 & 6.28 & 2.50 & 2.65 & 0.00 & 0.15 & 2.50 & 2.40 \\
\hline 19 & 3.75 & 3.88 & 0.00 & -0.14 & 2.50 & 2.80 & 5.00 & 4.92 & 0.00 & -0.09 \\
\hline 20 & 2.50 & 2.42 & 0.00 & -0.03 & 3.75 & 4.06 & 1.25 & 1.14 & 0.00 & -0.22 \\
\hline 21 & 5.00 & 4.83 & 0.00 & -0.09 & 1.25 & 1.41 & 3.75 & 3.77 & 0.00 & 0.11 \\
\hline 23 & 5.00 & 5.33 & 0.00 & -0.22 & 1.25 & 1.37 & 0.00 & 0.06 & 3.75 & 3.75 \\
\hline 24 & 1.25 & 1.38 & 0.00 & -0.10 & 5.00 & 5.09 & 0.00 & 0.10 & 2.50 & 2.52 \\
\hline 28 & 2.50 & 2.52 & 0.00 & -0.09 & 2.50 & 2.82 & 1.25 & 1.30 & 5.00 & 4.90 \\
\hline
\end{tabular}




\section{Determination of the maximum orders}

The reconstruction error decreases with the increase of the maximum orders $(q Q, n N$ and $m M$ ) and the change of reconstruction error was basic stable when $n N=3, m M=3$ and $q Q=4$.
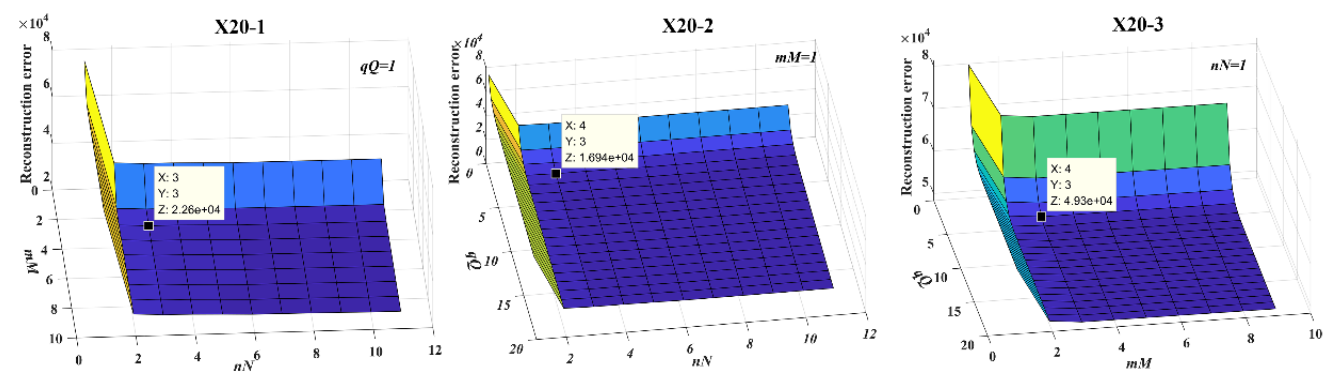

Figure S1 Reconstruction error for X20 dataset (1) x20-1: $q Q=1$, reconstruction error changes trend with order $n N$ and $m M$; (2) x20-2: $m M=1$, reconstruction error changes trend with order $n N$ and $q Q$; (3) $\mathrm{x} 20-3: n N=1$, reconstruction error changes trend with order $m M$ and $q Q$. 


\section{The modeling parameters of APQLD for the Data set I and Data set II}

Table S3. The performance of the APQLD models for Data I

\begin{tabular}{|c|c|c|c|c|c|c|c|c|c|c|c|}
\hline \multirow[t]{2}{*}{ Data set } & \multirow[t]{2}{*}{ Analyte } & \multirow[t]{2}{*}{ Variables } & \multicolumn{5}{|c|}{ Calibration } & \multicolumn{2}{|c|}{ Cross-validation } & \multicolumn{2}{|c|}{ Prediction } \\
\hline & & & $R_{c}^{2}$ & $R_{a d j}^{2}$ & $F$-test & $p$-Value & $R M S E_{c}$ & $R_{c v}^{2}$ & $R M S E_{c v}$ & $R_{p}^{2}$ & $R M S E_{p}$ \\
\hline \multirow[t]{2}{*}{$\mathrm{X} 20$} & Adrenaline & 1 & 0.9920 & 0.9918 & $5.84 \times 10^{3}$ & $5.86 \times 10^{-51}$ & 0.0289 & 0.9908 & 0.0304 & 0.9411 & 0.1028 \\
\hline & Noradrenaline & 2 & 0.9850 & 0.9843 & $3.08 \times 10^{3}$ & $1.70 \times 10^{-44}$ & 0.0430 & 0.9821 & 0.0455 & 0.9337 & 0.1386 \\
\hline \multirow[t]{2}{*}{ X60 } & Adrenaline & 1 & 0.9914 & 0.9912 & $5.42 \times 10^{3}$ & $3.34 \times 10^{-50}$ & 0.0300 & 0.9901 & 0.0315 & 0.9404 & 0.1032 \\
\hline & Noradrenaline & 2 & 0.9847 & 0.9841 & $3.03 \times 10^{3}$ & $2.48 \times 10^{-44}$ & 0.0434 & 0.9818 & 0.0459 & 0.9450 & 0.1379 \\
\hline \multirow[t]{2}{*}{ Xlarge } & Adrenaline & 2 & 0.9949 & 0.9947 & $9.13 \times 10^{3}$ & $1.71 \times 10^{-55}$ & 0.0234 & 0.9937 & 0.0252 & 0.9264 & 0.1151 \\
\hline & Noradrenaline & 2 & 0.9913 & 0.9909 & $5.34 \times 10^{3}$ & $4.79 \times 10^{-50}$ & 0.0328 & 0.9892 & 0.0353 & 0.8840 & 0.1947 \\
\hline
\end{tabular}


Table S4. The performance of the quantitative models obtained from APQLD method for Data II

\begin{tabular}{|c|c|c|c|c|c|c|c|c|c|c|c|}
\hline \multirow[b]{2}{*}{ Data set (size) } & \multirow[b]{2}{*}{ Analyte } & \multirow[b]{2}{*}{ Variables } & \multicolumn{5}{|c|}{ Calibration } & \multicolumn{2}{|c|}{ Cross-validation } & \multicolumn{2}{|c|}{ Prediction } \\
\hline & & & $R_{c}^{2}$ & $R_{a d j}^{2}$ & $F$-test & $p$-Value & $R M S E_{c}$ & $R_{\text {looccv }}^{2}$ & $R M S E_{c v}$ & $R_{p}^{2}$ & $R M S E_{p}$ \\
\hline \multirow{5}{*}{$\begin{array}{l}\text { NMR-EEM-LCMS } \\
(8297 \times 21 \times 3 \times 28)\end{array}$} & Val-Tyr-Val & 1 & 0.2928 & 0.2555 & 7.87 & 0.011 & 1.6923 & 0.1785 & 1.7474 & 0.0622 & 1.5609 \\
\hline & Trp-Gly & 2 & 0.7185 & 0.6872 & $4.85 \times 10$ & $1.23 \times 10^{-6}$ & 1.1797 & 0.6262 & 1.2661 & 0.8381 & 0.9894 \\
\hline & Phe & 1 & 0.9799 & 0.9788 & $9.24 \times 10^{2}$ & $1.41 \times 10^{-17}$ & 0.2668 & 0.9758 & 0.2787 & 0.989 & 0.3838 \\
\hline & Malto & 2 & 0.9920 & 0.9911 & $2.35 \times 10^{3}$ & $2.23 \times 10^{-21}$ & 0.1675 & 0.9893 & 0.1796 & 0.9982 & 0.1353 \\
\hline & Propanol & 2 & 0.9936 & 0.9929 & $2.96 \times 10^{3}$ & $2.55 \times 10^{-22}$ & 0.1781 & 0.9914 & 0.192 & 0.9943 & 0.2392 \\
\hline \multirow{5}{*}{$\begin{array}{l}\text { NMR-LCMS } \\
(8297 \times 8 \times 2 \times 28)\end{array}$} & Val-Tyr-Val & 1 & 0.3115 & 0.2753 & 8.60 & $8.60 \times 10^{-3}$ & 1.6697 & 0.2209 & 1.6971 & 0.2317 & 1.7259 \\
\hline & Trp-Gly & 0 & 0 & 0.0000 & 2.00 & $1.73 \times 10^{-1}$ & 2.1094 & 0.0087 & 2.1011 & $\mathrm{NaN}$ & 2.4622 \\
\hline & Phe & 1 & 0.5356 & 0.5112 & $2.19 \times 10$ & $1.63 \times 10^{-4}$ & 1.2808 & 0.4663 & 1.3137 & 0.1175 & 1.2026 \\
\hline & Malto & 2 & 0.9944 & 0.9929 & $3.34 \times 10^{3}$ & $7.97 \times 10^{-23}$ & 0.1491 & 0.9901 & 0.1731 & 0.9972 & 0.1069 \\
\hline & Propanol & 2 & 0.9753 & 0.9726 & $7.50 \times 10^{2}$ & $9.83 \times 10^{-17}$ & 0.3503 & 0.9621 & 0.4023 & 0.9734 & 0.3479 \\
\hline
\end{tabular}

